\title{
A SECOND OPINION ON RELATIVE TRUTH
}

\section{RAMIRO CASO}

Universidade de Buenos Aires

Departamento de Filosofia

Ciudad de Buenos Aires, Argentina

ramirocaso08@gmail.com

Received: 13.07.2015; Accepted: 21.09.2015

\begin{abstract}
In 'An undermining diagnosis of relativism about truth', Horwich claims that the notion of relative truth is either explanatorily sterile or explanatorily superfluous. In the present paper, I argue that Horwich's explanatory demands set the bar unwarrantedly high: given the philosophical import of the theorems of a truth-theoretic semantic theory, Horwich's proposed explananda, what he calls acceptance facts, are too indirect for us to expect a complete explanation of them in terms of the deliverances of a theory of meaning based on the notion of relative truth. And, to the extent that there might be such an explanation in certain cases, there is no reason to expect relative truth to play an essential, ineliminable role, nor to endorse the claim that it should play such a role in order to be a theoretically useful notion.
\end{abstract}

Keywords: truth relativism, radical relativism, truth-theoretic semantics.

\section{Introduction}

In (2014), Horwich argues that semantic theories based on the notion of relative truth are empirically useless, for they are unable to account for the phenomena any suitable theory of meaning ought to explain: certain observable facts concerning our linguistic activity that he calls acceptance (and rejection) facts. Moreover, even if this were not so and there were an adequate explanation of those phenomena in terms of relative truth, the notion of relative truth itself would be explanatorily idle. Put more bluntly, relative truth is either explanatorily sterile or explanatorily superfluous. In either case, truth-theoretic semantic theories based upon the notion of relative truth ought to be abandoned in favor of a use-theoretic theory of meaning.

Manuscrito - Rev. Int. Fil., Campinas, v. 38, n.2, pp.65-88, jul.-ago. 2015. 


\section{6凸amiro Caso}

In this paper, I'll essay a defense of relativistic theories of meaning against Horwich's criticism. It should be noted from the start that, although Horwich mounts his case against MacFarlane's way of spelling out a truthrelativistic theory of meaning ( $c f r$. MacFarlane (2014, ch. 3-4)), the criticism easily applies to any relativistic theory of meaning cast in the semantico-pragmatic style of Kaplan (1989). Hence, Horwich's objection affects more than a single brand of relativism. In order to discuss the objection, I'll start with a brief account of what relativistic theories of meaning are (section 2). Then, I'll move on to a consideration of Horwich's objection to theories of this kind, temporarily granting the assumption that theories of meaning ought to explain acceptance (and rejection) facts (section 3). Finally, I'll provide a reply to Horwich's claims (section 4), and I'll provide some reasons for rejecting acceptance (and rejection) facts as the proper explananda of a relativistic theory of meaning (section 5).

\section{Semantico-pragmatic theories based on relative truth}

The first order of business is to provide an adequate characterization of Horwich's targets, the semantico-pragmatic theories of meaning that deploy the notion of relative truth. As Horwich's objection is explicitly directed against MacFarlane's brand of relativism, I'll start with MacFarlane's characterization.

MacFarlane (2014) favors a purely semantic presentation of relativism (which justifies calling it truth relativism) according to which propositional truth is assessment sensitive, in the sense that the truth value of evaluative propositions (i.e., the propositions expressed by sentences such as 'Sushi is delicious' and 'Stealing is wrong', among others) partly depends on a context of assessment. The properly semantic part of a relativistic theory of meaning, which MacFarlane calls semantics proper, aims to define, in the style of Kaplan (1989), a predicate 'true at $\langle w, s\rangle^{\prime}$ for propositional contents, where $\langle w, s\rangle$ is a circumstance of evaluation composed of a possible world $w$ and an evaluative parameter $s$ (a standard of taste, a system of moral norms, etc.). After semantics proper comes post-semantics, the part of a theory of meaning whose job is to characterize a predicate of immediate pragmatic relevance, 'true as used at $c$ and as assessed from $c^{\prime}$ ', in terms of the properly semantic predicate 'true at $\langle w, s\rangle$ ', along the following lines: 
The proposition expressed by a sentence $S$ at a context $c$ is true as used at $c$ and as assessed from $c^{\prime}$ iff it is true at $\left\langle w_{c}, s_{c^{\prime}}\right\rangle$,

where $w_{c}$ is the world of $c$ and $s_{c^{\prime}}$ is the evaluative parameter relevant at the context of assessment $c^{\prime}$. This is a predicate of immediate pragmatic relevance in the sense that it provides the interface between the theoretical concept of truth at a circumstance for propositions and central pragmatic concepts. In particular, the interface with pragmatics comes by linking this predicate with speech acts such as assertion, retraction and rejection by means of principles like the following:

Reflexive Truth Rule. An agent in context $c$ is permitted to assert $p$ only if $p$ is true as used at $c$ and as assessed from $c$.

Retraction Rule. An agent in context $c^{\prime}$ is required to retract an (unretracted) assertion of $p$ made at $c$ if $p$ is not true as used at $c$ and as assessed from $c^{\prime}$.

Rejection Rule. An agent in context $c^{\prime}$ is permitted to reject an assertion of $p$ made at $c$ if $p$ is not true as used at $c$ and as assessed from $c^{\prime}$.

As we've already remarked, the Reflexive Truth Rule, the Retraction Rule and the Rejection Rule act as semantics-pragmatics bridging principles, for they connect the theoretical truth predicate 'true at $\langle w, s\rangle$ ' with normative conditions for the speech acts of assertion, retraction, and rejection, via a predicate with immediate pragmatic relevance, the propositional predicate 'true as used at $c$ and as assessed from $c^{\prime \prime}$.

Of course, other presentations are also possible. In the presentation I favor, relativism about truth —or, more properly, radical relativism- is a semantico-pragmatic approach to natural languages according to which the correctness of assertive utterances of sentences belonging to evaluative discourses is sensitive to the perspective from which those utterances are assessed. ${ }^{1}$ As before, the properly semantic part of radical relativism aims to define the predicate 'true at $\langle w, s\rangle$ ' for propositional contents. The pragmatic part contains certain principles linking truth at a circumstance for propositions with correctness for utterances-for example:

${ }^{1}$ See my (2014) for details.

Manuscrito - Rev. Int. Fil., Campinas, v. 38, n.2, pp.65-88, jul.-ago. 2015. 
Utterance correctness. An utterance of a sentence $S$ made at $c$ is correct, as assessed from $c^{\prime}$, if and only if the proposition expressed by $S$ at $c$ is true at $\left\langle w_{c}, s_{c^{\prime}}\right\rangle$,

where $w_{c}$ and $s_{c^{\prime}}$ are as before. Thus, Utterance correctness is a semanticspragmatics bridging principle linking the semantic-theoretic notion of truth at a circumstance of evaluation for propositions with a notion of immediate pragmatic relevance, correctness as assessed from a context $c^{\prime}$ for assertive utterances (cfr. also Kölbel $(2008 \mathrm{a}, 2008 \mathrm{~b}, 2009)$ for a similar articulation of a moderate version of relativism).

Be it under one style of semantico-pragmatic theorizing or the other, we should note the existence of a general theoretical schema that consists of a (properly) semantic-theoretic predicate 'true at $\langle w, s\rangle$ ' and a predicate with immediate pragmatic relevance, which allows for the connection between semantics and facts about our use of language. This is an important feature of relativistic theories of meaning, for Horwich's objection will crucially rely on the need for these principles in order to connect the semantic-theoretic predicate of truth at a circumstance with language use.

A final twist: since, for Horwich, the explananda of a semantic theory are facts about acceptance (and rejection) of sentences, we'll also need semantic and pragmatic concepts defined for sentences. The structure of a Kaplan-style semantic theory makes this easy, for the propositional truth predicate 'true at $<w$, $s>$ ' is actually introduced on top of the recursive definition of a sentential truth predicate, 'true at a context $c$ with respect to a circumstance of evaluation $<w$, $s>$ ', where $c$ is a context of utterance. More precisely, the propositional truth predicate is introduced by the following equivalence:

The proposition expressed by $S$ at $c$ is true at $\langle w, s\rangle$ iff $S$ is true at $c$ with respect to (w.r.t.) $\langle w, s\rangle$,

where the proposition expressed by $S$ at a context $c$ is (represented by) the function that maps a world-perspective pair $\langle w, s\rangle$ to truth just in case $S$ is true at $c$ with respect to $\langle w, s\rangle$, and to falsity otherwise. ${ }^{2}$ Now we may restate the

${ }^{2}$ Even more precisely, the predicate that is actually defined is 'true at a context $c$ with

Manuscrito - Rev. Int. Fil., Campinas, v. 38, n.2, pp.65-88, jul.-ago. 2015. 
bridging principles in terms of this last predicate:

Reflexive Truth Rule (for sentences). An agent in context $c$ is permitted assertively to utter $S$ only if $S$ is true at $c$ w.r.t. $\left\langle w_{c}, s_{c}\right\rangle$.

Retraction Rule (for sentences). An agent in context $c^{\prime}$ is required to retract an (unretracted) assertive utterance of $S$ made at $c$ if $S$ is not true at $c$ w.r.t. $\left\langle w_{c}, s_{c^{\prime}}\right\rangle$.

Rejection Rule (for sentences). An agent in context $c^{\prime}$ is permitted to reject an assertive utterance of $S$ made at $c$ if $S$ is not true at $c$ w.r.t. $<w_{c}$, $s_{c^{\prime}}>$.

Utterance correctness (for sentences). An utterance of a sentence $S$ made at $c$ is correct, as assessed from $c^{\prime}$, if and only if $S$ is true at $c$ w.r.t. $\left\langle w_{c}, s_{c^{\prime}}\right\rangle$.

We are now in a position to address Horwich's objection.

\section{Horwich on relative truth}

As we've already advanced, in (2014) Horwich attempts to argue that either relativistic theories of meaning are unable to explain acceptance/rejection facts, or, to the extent that they are able to do so, relative truth is doing no real, explanatory work and is, therefore, a superfluous concept that we ought to reject.

As the starting point of his case against relativism, Horwich notes that a set of truth-theoretic semantic axioms can have the required empirical import only if supplemented with principles linking the truth conditions assigned to the sentences of the language under study with facts about our use of language. This is true, particularly, of the ways in which we've presented relativistic theories of meaning, for the role of the semantics-pragmatics bridging principles we've identified is precisely that of linking the distribution of truth values at the different circumstances of evaluation specifiable in the properly semantic part of a relativistic theory of meaning with facts about the correctness or incorrectness of the corresponding assertive utterances. It is through the

respect to a circumstance of evaluation $\langle w, s\rangle$, under an assignment $f$ for formulas. We are dropping the assignment in order to make things more legible.

Manuscrito - Rev. Int. Fil., Campinas, v. 38, n.2, pp.65-88, jul.-ago. 2015. 


\section{$70 \llbracket$ Ramiro Caso}

specification of correctness conditions for assertive utterances that these principles have normative consequences concerning the acceptance and rejection of utterances, as well as concerning assertion and retraction. (Obviously, this is why Horwich's objection will apply equally to both kinds of presentation, for both share this feature: properly semantic notions have empirical import only insofar as they are connected with pragmatics by means of this kind of principles.)

Now, Horwich raises the stakes at this point, by insisting that the empirical consequences of a semantic theory should be statements about linguistic activity: the connection between semantic theory and language use should be between distributions of truth values at different circumstances and concrete and observable facts about our linguistic activity. These are basically what Horwich (2010, ch. 8) calls acceptance facts (and we could —and shouldinclude facts about rejection as well), i.e., facts about acceptance (and rejection) of sentences by particular speakers on given situations. Certainly, at this point, the advocate of relativism might be tempted to halt Horwich's considerations by holding that the empirical basis of a semantic theory (at least for the quarters of truth-theoretic semantics) is usually thought to consist of the intuitive judgments competent speakers make regarding the truth conditions of sentences in context, or concerning the (objective) correctness conditions of the corresponding utterances-so that we should not grant that the explananda are concrete, observable cases of acceptance and rejection. However, certain considerations recommend letting Horwich's objection roll, at least pro tem, and consider the possibility of coming up with an explanation of actual linguistic activity in terms of the deliverances of a truth-theoretic semantic theory: as is well known, intuitive judgments may not be as stable and as clear as desirable; and the careful consideration of Horwich's requirement to account for actual linguistic practice will allow us to conclude that intuitive judgments (their relative lack of stability and clarity notwithstanding) constitute an empirical basis for semantic theories that is more immediate than acceptance/rejection facts, for the explanation of these forces us to take into consideration a wider set of theories.

So, what are the principles that should make the connection between truth-theoretic axioms and theorems and the use of language? Since the facts to explain are facts about acceptance and rejection, the natural candidates are the norms that guide the speech acts we normally use in performing those activities. We've already seen the norms for assertion, retraction and rejection. To these we 
should add the norm for acceptance:

Acceptance Rule (for sentences). An agent in context $c^{\prime}$ is permitted to accept an assertive utterance of $S$ made at $c$ only if $S$ is true at $c$ w.r.t. $<w$, $s_{i^{\prime}}>$.

For Horwich, these principles have the burden of providing the connection between the semantic theorems and the use of language. But how might they do it?

A problem that Horwich immediately points out is that these principles actually do not allow us to explain any linguistic activity at all, for normative principles like these cannot effectively constrain what we actually do. That is, from the fact that we have, e.g., permission assertively to utter or to accept a sentence only if it is true, or the obligation to retract its assertive utterance if it is false, it doesn't follow that we actually do so. Thus, for Horwich, what would have the required import are not those principles themselves, but that we had a tendency or propensity to abide by those principles. Given such a propensity, the purely semantic theorems that constitute the deliverances of the truth-theoretic axioms would allow us to explain the acceptance/rejection facts.

Horwich (2010, ch. 8) is quite explicit about how such a tendency could help in explaining linguistic activity in the case of acceptance facts, though what he says easily applies to rejection facts as well. As we've already seen, acceptance facts are concrete cases in which a speaker accepts a certain sentence. As it turns out, sentence acceptance is somewhat complex, and encompasses two cases: one might accept a sentence by assertively uttering it, or by accepting someone else's utterance of that sentence. ${ }^{3}$ In the first case, the primary target of acceptance is the sentence itself (or the sentence as used and as assessed from the context of assertion), whereas in the second case, the primary target of acceptance is the utterance (or the sentence as used at its original context and as assessed from the context at which the acceptance takes place). Thus, acceptance facts are facts about speakers uttering sentences in certain circumstances, and about speakers accepting others' utterances in certain circumstances. In the spirit of truth relativism we could (and should) add rejection facts to acceptance facts. These

\footnotetext{
${ }^{3}$ Three (or four) if we allow for conditional acceptance-the kind of acceptance related to inferential behavior. However, there is no need for this complication here.
}

Manuscrito - Rev. Int. Fil., Campinas, v. 38, n.2, pp.65-88, jul.-ago. 2015. 


\section{2『Ramiro Caso}

also come in two varieties: the rejection of someone else's assertive utterance, and the retraction of one's own assertive utterances. So, the linguistic activity to be explained also includes concrete cases of rejection and concrete cases of retraction. (Without forgetting these nuances, I'll speak, for the time being, simply of acceptance and rejection facts.)

Horwich $(2010$, ch. 8$)$ is quite explicit about the form of a possible explanation for acceptance facts, a form of explanation that makes it clear in which sense the alleged tendency to abide by the principles governing assertion and acceptance plays a central role in the explanation of linguistic activity. It should be noted that Horwich gives an explicit formulation for the case of truthconditional semantics based on the notion of absolute truth in the semantic style of Davidson (1967). However, what he says can be transposed to a semantic theory based on the notion of relative truth and couched in the semantic style we've chosen.

Let's start with Horwich's criticism as it applies to a Davidsonian semantic theory. In (2010, ch. 8), Horwich holds that Davidsonian truth conditional semantics would be incapable of explaining acceptance facts because there wouldn't be any causal-explanatory link between the (alleged) semantic fact that a sentence has certain truth conditions and the fact that, in particular circumstances, an agent accepts that sentence. That is, truth-conditional semantics wouldn't be able to predict, with high enough probability, that a given acceptance fact will take place, on the basis of the truth conditions of the accepted sentence.

Thus, we start to see what form an explanation of an acceptance fact should take: it should employ an alleged causal-explanatory link between the possession of certain truth conditions by the accepted sentence and its acceptance by an agent under certain circumstances in order to assign a high enough probability to the occurrence of a particular case of acceptance in circumstances of that type. The explanatory schema that Horwich deems initially plausible is quite illustrative of this point. For him, an explanation of an acceptance fact could take the form of the following derivation:

1. $S$ is true iff $p$

2. $A$ will probably accept $S$ iff $S$ is true

3. $A$ will probably accept $S$ iff $p$

4. $p$

Manuscrito - Rev. Int. Fil., Campinas, v. 38, n.2, pp.65-88, jul.-ago. 2015. 
5. $A$ will probably accept $S$

In this derivation, premise 1 is provided by the proposed truth-conditional analysis of sentence $S$. Premise 2 captures the alleged propensity to abide by the norm for sentence acceptance (i.e., the alleged propensity to accept $S$ just in case $S$ is true); 3 follows from 1 and 2; 4 results from observation, or from some other way of determining that a truth condition for $S$ actually takes place; 5 is just the conclusion that $A$ will probably accept $S$, given its truth, in the circumstances she's in. If we pause to check this derivation, it is easy to see that the crucial step (indeed, the step that offers the causal-explanatory link required by Horwich) is step 2, the step that expresses the assumption that we have a tendency to abide by the normative requisite for sentence acceptance.

In the case of rejection, a similar schema could be provided:

1. $S$ is true iff $p$

2. $A$ will probably reject $S$ iff $S$ is not true

3. $A$ will probably reject $S$ iff not- $p$

4. not- $p$

5. $A$ will probably reject $S$

Here, the crucial step linking the semantic analysis of sentence $S$ with a concrete fact of rejection of $S$ by $A$ is step 2 again, that is, the step that expresses the alleged tendency to reject a sentence just in case it is false.

The problem for this line of thought, of course, is that we do not have the alleged tendencies. That is, we do not have a tendency to accept a sentence just in case it is true, and we do not have a tendency to reject a sentence just in case it is false. Thus, the pretension of explanatory adequacy of truth-conditional semantics seems to crash at this point.

This is particularly clear in the case of non-evaluative sentences, such as:

(1) Snow is white.

(2) Red soils have a high concentration of iron.

If we actually had those tendencies, then our epistemic lives would be considerably happier than they actually are. The consideration of evaluative 


\section{$74 \llbracket$ Ramiro Caso}

sentences such as:

(3) Sushi is delicious,

on the other hand, might generate the hope that a semantic theory based on the notion of relative truth may succeed where one based on the notion of absolute truth fails. Of course, even so, relativistic theories of meaning would end up being inadequate, since they yield, for non-evaluative sentences, the same results as non-relative truth-theoretic semantic theories. Thus, insofar as a theory of meaning based on the notion of relative truth should also account for nonevaluative sentences, it would have the same fate as semantic theories based on the notion of absolute truth. However, relativism would be adequate at least for evaluative sentences, that is, those sentences that motivate the truth-relativistic approach in the first place. The reason is the following: even though it is implausible to suggest that we have a tendency to accept (reject) non-evaluative sentences just in case they are true (false), it might be plausible to claim that we have a tendency to accept evaluative sentences just in case they are true (false) as assessed from our own perspectives.

Following this line of thought, Horwich (2014) points out that, in the case of evaluative sentences, the following principles have certain plausibility:

(4) At a context $c, A$ will probably assertively utter $S$ just in case $S$ is true at $c$ w.r.t. $\left\langle w_{c}, s_{c}\right\rangle$.

(5) At a context $c^{\prime}, A$ will probably accept an assertive utterance of $S$ made at $c$ just in case $S$ is true at $c$ w.r.t. $\left\langle w_{c}, s_{c^{\prime}}\right\rangle$. ${ }^{4}$

Again, these principles might serve as a link between the (alleged) semantic fact

\footnotetext{
${ }^{4}$ Actually, Horwich considers an undifferenciated principle for sentence acceptance:

At a context $c, A$ will probably accept $S$ just in case $S$ is true in $A$ 's context of assessment.

(cfr. Horwich $(2014,745)$.) However, once we take into account the difference between assertion and acceptance, we need to provide two different principles. Also, relativity to a context of utterance is required in order to make room for indexicality, as well as for non-indexical dependence on features of the context of utterance, such as the world of that context.
}

Manuscrito - Rev. Int. Fil., Campinas, v. 38, n.2, pp.65-88, jul.-ago. 2015. 
that an evaluative sentence has certain truth conditions relative to an evaluative perspective, and the concrete, observable fact of its acceptance by an agent on a particular occasion, since they express the tendency to accept an evaluative sentence just in case it is true relative to one's own evaluative perspective. Similar principles for rejection and retraction,

(6) At a context $c^{\prime}, A$ will probably reject an assertive utterance of $S$ made at $c$ just in case $S$ is not true at $c$ w.r.t. $\left\langle w_{c}, s_{c^{\prime}}\right\rangle$.

(7) At a context $c^{\prime}, A$ will probably retract an (unretracted) assertive utterance of $S$ made at $c$ just in case $S$ is not true at $c$ w.r.t. $\left\langle w_{c}, s_{c^{\prime}}\right\rangle$,

would allow us to link semantic facts with concrete, observable cases of rejection and retraction.

Assuming the plausibility of these principles, we could have the hope of being able to explain acceptance and rejection facts by means of derivations analogous to the one explicitly given by Horwich. ${ }^{5}$ If we distinguish clearly between acceptance and assertion, on the one hand, and rejection and retraction, on the other, it is possible to come up with four schemata for explaining acceptance and rejection facts:

\footnotetext{
${ }^{5}$ These principles don't seem to be plausible even when restricted to evaluative sentences, for there are evaluative questions whose decision might be quite complex-just think about the numerous factual considerations that may be relevant in order to determine whether a given action is morally wrong, or whether a given belief is justified, and about all the possible interactions and incompatibilities between moral or epistemic norms or policies of different levels that constitute moral and epistemic systems. Maybe these principles are plausible only for evaluative properties that are simple from the factual and normative point of view, such as those involved in matters of taste, matters of humor, etc. In these cases, given the simple character of the normative side of the judgment, first hand knowledge of the facts of the disputed question (e.g., knowing how sushi tastes) might be sufficient to know everything there is to know in order to decide the corresponding evaluative question (e.g., whether sushi is delicious or not). However, I'll keep the assumption that these principles are plausible for evaluative sentences in general, since my answer to Horwich's concerns won't depend on any take on this issue.
}

Manuscrito - Rev. Int. Fil., Campinas, v. 38, n.2, pp.65-88, jul.-ago. 2015. 


\section{6[Ramiro Caso}

\section{Sentence acceptance (assertion)}

1. For all $S, c, w, s: S$ is true at $c$ w.r.t. $\langle w, s>$ iff the proposition expressed by $S$ at $c$ is true at $\langle w, s\rangle$

2. A subject $A$ at a context $c$ will probably assertively utter $S$ iff $S$ is true at $c$ w.r.t. $\left\langle w_{c}, s_{c}\right\rangle$

3. $A$ will probably assertively utter $S$ iff the proposition expressed by $S$ at $c$ is true at $\left\langle w_{c}, s_{c}\right\rangle$

4. The proposition expressed by $S$ at $c$ is true at $\left\langle w_{c}, s_{c}\right\rangle$

5. $A$ will probably assertively utter $S$

\section{Utterance acceptance}

1. For all $S, c, w, s: S$ is true at $c$ w.r.t. $\langle w, s\rangle$ iff the proposition expressed by $S$ at $c$ is true at $\langle w, s\rangle$

2. A subject $A$ at a context $c^{\prime}$ will probably accept an assertive utterance $u$ of $S$ made at $c$ iff $S$ is true at $c$ w.r.t. $\left\langle w_{c}, s_{c^{\prime}}\right\rangle$

3. $A$ will probably accept $u$ iff the proposition expressed by $S$ at $c$ is true at $\left\langle w_{c}, s_{c^{\prime}}\right\rangle$

4. The proposition expressed by $S$ at $c$ is true at $\left\langle w_{c}, s_{c^{\prime}}\right\rangle$

5. $A$ will probably accept $u$

\section{Utterance rejection}

1. For all $S, c, w, s: S$ is true at $c$ w.r.t. $\langle w, s\rangle$ iff the proposition expressed by $S$ at $c$ is true at $\langle w, s\rangle$

2. A subject $A$ at a context $c^{\prime}$ will probably reject an assertive utterance $u$ of $S$ made at $c$ iff $S$ is not true at $c$ w.r.t. $\left\langle w_{c}, s_{c^{\prime}}\right\rangle$

3. $A$ will probably reject $u$ iff the proposition expressed by $S$ at $c$ is not true at $\left\langle w_{c}, s_{c}\right\rangle$

4. The proposition expressed by $S$ at $c$ is not true at $\left\langle w_{c}, s_{c}\right\rangle$

5. A will probably reject $u$ 


\section{Retraction}

1. For all $S, c, w, s: S$ is true at $c$ w.r.t. $\langle w, s>$ iff the proposition expressed by $S$ at $c$ is true at $\langle w, s\rangle$

2. A subject $A$ at a context $c^{\prime}$ will probably retract an (unretracted) assertive utterance $u$ of $S$ made at $c$ iff $S$ is not true at $c$ w.r.t. $\left\langle w_{c}, s_{c^{\prime}}\right\rangle$

3. $A$ will probably retract $u$ iff the proposition expressed by $S$ at $c$ is not true at $\left\langle w_{c}, s_{c^{\prime}}\right\rangle$

4. The proposition expressed by $S$ at $c$ is not true $\left\langle w_{c}, s_{c}\right\rangle$

5. $A$ will probably retract $u$

Through these schemata, the distributions of truth values relative to circumstances of evaluation posited by a truth-relativistic semantic theory, together with the tendencies to abide by the norms for assertion, acceptance, rejection and retraction - when restricted to evaluative sentences-, would be enough to explain acceptance and rejection facts concerning evaluative sentences.

However, Horwich continues, these principles are not really illuminating of acceptance and rejection facts, for the explanations remain silent about what it is for a sentence to be true (or false) at a context of use and a context of assessment, and nothing is said about what it is for a context to be a context at which an evaluative sentence is true (or false, as the case may be). Thus, in order to have a full explanation of acceptance and rejection facts, we also have to make explicit the implications of certain non-semantic features of the contexts of use and assessment for the possession of one or other truth value by an evaluative sentence relative to those contexts. That is, besides citing principles linking relative truth and the use of language, we are owed some principles linking nonsemantic features of context with the notion of relative truth. That is, we must answer the question: What it is for a context to be a context at which an evaluative sentence is true (or false)?

Now, once we do this and we make explicit what is involved in a sentence's being true relative to a context of assessment, it becomes clear that any deployment of the notion of relative truth in the explanation of acceptance and rejection facts is, at best, accessory. Indeed, e.g., to the question, "What it is for a context to be a context at which "Sushi is delicious" is true?", the answer is: It is to be a context such that its agent likes the taste of sushi. That is, the 


\section{8[Ramiro Caso}

following principle will hold:

(8) 'Sushi is delicious' is true at $c$ w.r.t. $\left\langle w_{c}, s_{c}\right\rangle$ iff the agent of $c$ likes the taste of sushi (at $t_{c}$ and $w_{c}$ ).

Now let's consider the alleged explanation of an acceptance fact of this sentence. Such an explanation would follow one of the schemata deployed above:

1. 'Sushi is delicious' is true at $c$ w.r.t. $\langle w, s\rangle$ iff the proposition expressed by 'Sushi is delicious' at $c$ is true at $\langle w, s\rangle$

2. A subject $A$ at a context $c$ will probably assertively utter 'Sushi is delicious' iff 'Sushi is delicious' is true at $c$ w.r.t. $\left\langle w_{c}, s_{c}\right\rangle$

3. $A$ will probably assertively utter 'Sushi is delicious' iff the proposition expressed by 'Sushi is delicious' at $c$ is true at $\left\langle w_{c}, s_{c}\right\rangle$

4. The proposition expressed by 'Sushi is delicious' at $c$ is true at $\left\langle w_{c}\right.$, $\left.s_{c}\right\rangle$

5. A will probably assertively utter 'Sushi is delicious' at $c$

It's clear that we can replace the right hand side of step 2 with the right hand side of (8), so as to obtain:

2'. A subject $A$ at a context $c$ will probably assertively utter 'Sushi is delicious' iff $A$ likes the taste of sushi (at $t_{c}$ and $w_{c}$ )

Thus, it is clear that the explanatory work is being done by the non-semantic features of the context at which the acceptance takes place, not by the sentence being true relative to that context: once we arrive at 2', any deployment of the notion of relative truth in the explanation of an acceptance fact becomes eliminable in terms of the non-semantic features of context. (And the same holds for the other varieties of acceptance and rejection.)

In this way, Horwich's dilemma for truth relativism is set: either a semantic theory based on the notion of relative truth is incapable of explaining acceptance and rejection facts (in the case of non-evaluative sentences), or the notion of relative truth plays an inessential role and can be eliminated (in the case of evaluative sentences). In either case, we should simply abandon the notion of relative truth. 
To this we could add the following consideration. The attentive reader will have already noticed two things. First, that steps 2 and 3, in each of the proposed schemata, are unnecessarily strong: all we need, in each case, is the right-to-left direction of the biconditional. Second, only an alleged tendency to abide by the normative principle for retraction would allow us to obtain this direction. The alleged tendency to abide by the normative principles for acceptance and assertion, on the other hand, would only vindicate the left-toright direction. This is due to the fact that the normative principle for retraction offers sufficient conditions for that action to be mandatory, whereas the normative principles for assertion and acceptance only offer necessary conditions for acceptance and assertion to be permissible. As for the principle for rejection, it provides a sufficient condition for rejection to be permissible, not mandatory, so any alleged tendency to abide by it wouldn't quite explain linguistic facts concerning rejection either. Thus, even if we had the alleged tendencies, most acceptance and rejection facts would still be quite hard to explain. So, it seems that relativism is in pretty bad shape.

\section{Semantic theory and linguistic activity}

Fortunately, it's not necessary to accept Horwich's objection. In order to see this, it should be noticed that the dilemma that Horwich presents to the relativist rests on four claims:

1. A semantic theory must explain (i.e., assign high probability to) the occurrence of concrete facts of acceptance and rejection.

2. In the case of a semantic theory based upon the notion of truth (be it absolute or relative), the explanation must exploit a causal-explanatory link between the possession of certain truth conditions on the part of a given sentence and the concrete facts of acceptance and rejection involving it. 3. In most cases, there is no causal-explanatory link between the possession of certain truth conditions by a given sentence and the corresponding acceptance/rejection facts

4. In those cases in which there is such a causal-explanatory link, what is really performing the explanatory work is a non-semantic feature of the context in which the acceptance/rejection takes place, not a semantic fact 


\section{0[Ramiro Caso}

about that sentence.

Horwich's considerations regarding the inexistence (in most cases) of a propensity to abide by the normative principles that guide acceptance, rejection, assertion and retraction seek to provide support to claim 3, and claim 4 receives its support, e.g., from the observation that what would actually explain a sincere acceptance of a sentence like 'Sushi is delicious' by a speaker at a given context would be the non-semantic fact that the speaker likes the taste of sushi, not the semantic fact that said sentence is true at that same context.

How may we resist Horwich's dilemma? Well, premise 1 seems suspicious, for it's not clear that an explanation of an event must require an assignment of high probability of occurrence to it. Simplifying a bit, a probabilistic prediction is considered explanatory when it assigns, to a certain event, a probability of taking place that is in the vicinity of an observed frequency for that event. If the observed frequency is low (and this, as is well known, depends, among other things, upon the way in which the event is described), a good probabilistic explanation of this event will be one that assigns to it a low probability of taking place. Thus, it's not obvious that we should grant claim 1, at least without a caveat. However, I'm prepared to grant it for the sake of argument.

Now, what about claim 2? Are there any good reasons to accept it? The answer seems to be, decidedly, no. In order to understand why, we should consider, in a more detailed manner, the theoretical role played by the theorems of a semantic theory based on the notion of truth (be it relative or not).

Allow me to start with semantic theories based upon the notion of absolute truth, since here the point is best appreciated. In its Davidsonian presentation, the goal of a semantic theory is recursively to assign truth conditions to the (declarative) sentences of a given language. Thus, the theorems of such a semantic theory associate sentences with truth-conditions in a formulation that, with some simplification, adopts a familiar form:

(9) 'Snow is white' is true iff snow is white.

(10) An occurrence of 'Snow is white' at a context $c$ is true iff snow is white (at the world of $c$ ).

Now, it should be kept in mind that these theorems, even if they do not employ

Manuscrito - Rev. Int. Fil., Campinas, v. 38, n.2, pp.65-88, jul.-ago. 2015. 
the notion of meaning in their formulation, are put forward as theoretical articulations of the meaning of sentences-or, more properly, as a theoretical articulation of that dimension of sentential meaning that is responsible for the objective correctness of the corresponding utterances. Thus, the goal of these theorems is to capture (employing a vocabulary that is, allegedly, better understood than, or otherwise susceptible of a more systematic treatment than, intensional vocabulary) those facts about the meaning of sentences that, more informally, could be captured by means of clauses like:

(11) 'Snow is white' (as uttered in c) means that snow is white. ${ }^{6}$

Essentially the same holds for those semantic theories whose goal is recursively to assign entities encapsulating truth conditions to sentences, such as propositions. These theories seek to prove theorems that, informally, may be formulated along the following lines:

(12) 'Snow is white' expresses the proposition that snow is white.

(13) 'Snow is white' (as used at $c$ ) expresses the proposition that snow is white.

Again, the idea behind these theorems is to capture, in a systematic way, the dimension of sentence meaning responsible for the objective correctness of the corresponding assertive utterances.

And the same holds for the way in which relativism has been articulated: the recursive definition of 'true at $c$ w.r.t. $\langle w, s\rangle$ ' for sentences (and of 'true at $\langle w, s\rangle$ ' for propositions) seeks to articulate the dimension of sentence meaning responsible for the objective correctness of the corresponding assertive utterances. This might be obscured by the fact that the truth definition achieves this systematization only when supplemented with an appropriate bridging principle. In any event, with such a principle at play, the theorems of the properly semantic part of the theory of meaning may also be seen as specifying and systematizing those facts about sentence meaning.

Now, if this is the philosophical content that we ought to read into the

${ }^{6}$ For a way to derive statements about meaning from statements about truth conditions, see Kölbel (2001).

Manuscrito - Rev. Int. Fil., Campinas, v. 38, n.2, pp.65-88, jul.-ago. 2015. 


\section{2[Ramiro Caso}

theorems of a truth-based semantic theory, then it is to be expected that they play a role in the explanation of actual linguistic activity. After all, the fact that our words and sentences mean what they do plays an important part (even though it is usually taken for granted) of why we utter them. (Just to belabor an obvious point, the fact that 'I'm hungry' means, on an approximate account, that the speaker is hungry — and not that she is bored - partially explains why a hungry speaker utters that sentence. As usual, the air of triviality is dispelled, and the explanatory role of facts about meaning is highlighted, when we deal with a sentence that belongs to a language different from the one in which the explanation is offered: the fact that the Italian sentence 'Ho fame' means that the speaker is hungry partially helps to explain why a hungry speaker utters that sentence on a particular occasion.) However, this role theorems play is quite restricted: they allow us to explain why a speaker utters a particular sentence and not a different one, but only if we already have an explanation of why she considered it relevant to express the corresponding proposition; in a similar way, they allow us to explain why she accepted or rejected a given utterance, but only if we already have an explanation of why she considers acceptable or unacceptable the proposition expressed by that utterance.

To put it differently, the semantic fact that a sentence means what it means (that it has certain truth conditions, that it expresses certain proposition) plays a role in the explanation of linguistic activity, but the burden of the explanation doesn't fall upon it, but upon an explanation of a different kind. In the case of the assertive utterance of a given sentence, it might be an explanation of why the speaker considered it relevant to make that claim instead of a different one (or instead of remaining silent). In the case of the acceptance of someone else's utterance, it might be an explanation of why she deemed acceptable what was said by means of that utterance. In the case of rejection, why it ought to be rejected. And in the case of retraction, why she considered that what was said by means of her earlier assertion ought to be now rejected, and why she deemed it relevant to make explicit her rejection. In all these cases, the explanation has an ineliminable epistemic component: it is necessary to explain why an agent considers certain proposition as true or false, or why she considers that there are other good reasons (e.g., appropriate or inappropriate evidence) to grant approval or to refrain from assenting. And there is also an ineliminable component of conversational rationality: it is necessary to explain why, independently of the epistemic evaluation, the agent deemed it relevant, or appropriate, to make the 
claim or retract it, rather than remaining silent, to accept or reject a claim instead of just letting it go through. Thus, the explanation of acceptance/rejection facts requires considerations that by far outreach what can be plausibly demanded of a truth-theoretic semantic theory, for they pertain to general issues having to do with epistemic and conversational rationality. This is why Horwich's second claim is not a plausible thesis: the link between semantic facts and actual linguistic activity is too mediated by epistemic and pragmatic considerations so as to think in the existence of a causal-explanatory route from those facts to this activity that allows us to assign, on its own, a high probability to concrete facts of acceptance and rejection.

What about claims 3 and 4? I think that they can (and should) be accepted. However, this acceptance is now far from being problematic. In the context of Horwich's reasoning, the acceptance of 3 generated the first horn of the dilemma: the inexistence of a causal-explanatory route mentioned in 3 , in the presence of claim 2, lead to the conclusion that a semantic theory based on the notion of relative truth is incapable of explaining facts about sentence acceptance and rejection; now, once we reject the idea that an explanation of acceptance/rejection facts ought to exploit such a causal-explanatory route (an idea that places an unmeetable explanatory demand on relativism alone), accepting claim 3 presents no problem.

Regarding 4, the claim responsible for the second horn of the dilemma, its acceptance should have never been regarded as problematic. The reason is the following. Let's consider an assertive utterance of:

(14) Sushi is delicious,

made by $A$ at a context $c$. In the style of explanation favored by Horwich, the attempt to explain this utterance in semantic terms relies on a clause like the following:

(15) At a context $c, A$ will probably assertively utter 'Sushi is delicious' iff 'Sushi is delicious' is true at $c$ w.r.t. $\left\langle w_{c}, s_{c}\right\rangle$.

Now, given that 'Sushi is delicious' is true at $c$ w.r.t. $\left\langle w_{c}, s_{c}\right\rangle$ just in case $A$ likes the taste of sushi, what is actually doing the explanatory work seems to be: 


\section{4[Ramiro Caso}

(16) At a context $c, A$ will probably assertively utter 'Sushi is delicious' iff $A$ likes the taste of sushi (at $w_{c}$ and $t_{c}$ ).

Does it follow from this that the semantic fact that (14) is true at $c$ w.r.t. $\left\langle w_{c}, s_{c}\right\rangle$ is eliminable in favor of an explanation that relies only on non-semantic facts in its place? Yes, but this, on itself, doesn't constitute a problem, since there is a sense in which (15) and (16) say the same thing, for they cite the same fact in explaining $A$ 's tendency to assertively utter (14) on that particular occasion: the difference between them is just that, while (15) describes that fact in a formal mode of speaking, (16) describes that same fact in a material mode of speaking. Thus, an explanation involving (15) and an explanation involving (16) would accomplish the same thing, e.g., explain an assertive utterance of the sentence 'Sushi is tasty' by an agent $A$ in context $c$, by citing the same fact described in different ways.

So, it is true that explanations invoking semantic facts can be eliminated in favor of explanations invoking non-semantic facts. But this doesn't mean that relative truth is an idle notion: it just provides a different way of describing the fact that the agent of the context likes the taste of sushi. And it should also be remarked that the usefulness of the predicate 'true at $c$ w.r.t. $\langle w, s\rangle$ ' (and of the propositional predicate 'true at $\langle w, s\rangle$ ') doesn't lie in the introduction of a substantial property that would be explanatorily ineliminable, but in allowing for the systematization of facts about sentential meaning. So, even though truth talk is strictly eliminable in the explanations devised by Horwich, we were wrong in expecting it not to be. Something similar applies to the predicates with immediate pragmatic relevance, 'true as used at $c$ and as assessed from $c^{\prime}$ ' in the case of MacFarlane's view of truth relativism, and 'correct as assessed from $c$, in the case of the other way of spelling out radical relativism: they do not introduce substantial, potentially analyzable properties that could feature in explanations in an essential, ineliminable way, but they function as bridges that allow for the connection between semantics proper and normative facts concerning the use of language. So requiring that these predicates be ineliminable in a putative explanation of acceptance and rejection facts would be going beyond the explicit purposes for which these predicates are introduced in a relativistic theory of meaning. 


\section{Conclusion}

In the previous section, I argued against Horwich's objection by pointing out that, given the philosophical content that truth-theoretic semantic theorems are supposed to have, they are supposed to play a role in the explanation of facts of acceptance and rejection, but only a restricted one, equivalent to the role played by the fact that a sentence means what it means (and not something else) in an explanation of why someone accepts it (or rejects it) on a given occasion. Thus, we shouldn't really expect the existence of a causal-explanatory route between facts about sentential meaning and facts of acceptance and rejection, at least in the following sense: a causal link between the truth or falsity of a sentence and a particular case of acceptance or rejection, such that the probability of someone accepting, or rejecting, a sentence $S$, given that $S$ is true, or false, is high (or high enough). Indeed, considerations regarding the epistemic or doxastic life of the agent are relevant, as well as considerations concerning conversational rationality. So we shouldn't grant Horwich's assumption that acceptance and rejection facts are the explananda of a theory of meaning, at least in the sense that considerations pertaining the theory of meaning alone - that is, independently of epistemic and pragmatic considerations - should be enough to assign high probability to particular events of acceptance and rejection, on the assumption that the corresponding sentences are true, in the case of acceptance, or false, in the case of rejection.

This much is obvious from the point of view of truth-theoretic semantics: given an explanation of what a sentence means, what is relevant for explaining a fact of acceptance (rejection) on those grounds is not whether that sentence is true (false), but whether the agent thinks it is true (false), or whether she thinks there are good grounds for accepting (or rejecting) it — and whether she thinks it is convenient to voice her acceptance (rejection) or to remain silent. Now, Horwich obviously knows this: his insistence on acceptance and rejection facts being the explananda of a theory of meaning stems from the requirement that semantics be an empirical science. After all, if semantics is supposed to be empirical, shouldn't it deal with the observable? The considerations we've developed thus far, on the other hand, point towards taking intuitions concerning objective correctness and intuitions concerning normative consequences for speech acts as being the evidential basis for a semantic theory, thus relegating the evidential role of acceptance and rejection facts. So, it seems that we may reject

Manuscrito - Rev. Int. Fil., Campinas, v. 38, n.2, pp.65-88, jul.-ago. 2015. 


\section{6[Ramiro Caso}

Horwich's acceptance and rejection facts as the explananda of a theory of meaning only by rejecting the claim that semantics is an empirical discipline.

This is not the place to tackle such a complex issue as that of the sense in which a semantic theory might be empirical, even if grounded upon speakers' intuitions concerning the objective correctness of assertive utterances. A consideration that may ameliorate the situation might be the following: speakers' intuitions are usually revealed by means of verbal activity, so being in agreement with intuition might count as explaining certain facts about our use of language - certainly, a highly specific use of language, but a possible use nonetheless. However, a consideration that is more relevant to Horwich's take on the empirical basis for a theory of meaning is the following: from the point of view of truth-theoretic semantics, a semantic theory is part of a cluster of theories which, together, have empirical consequences concerning linguistic activity.

As we've already remarked, in order to explain an acceptance (rejection) fact, we have to take into account what the sentence object of acceptance (rejection) means, why the speaker had reasons to think that the sentence was true (false) or otherwise warranted (unwarranted), why he deemed it convenient/relevant to voice her view instead of remaining silent, and more generally, if insincere, why she decided to reject a sentence she considered true or warranted, or why she decided to accept a sentence she considered false or unwarranted, etc.

In order to drive the point home, let's consider two cases, one of acceptance, one of rejection. In the first case, $A$ utters 'I'm hungry'. In the second case, $A$ rejects $B$ 's utterance of that same sentence, by tokening, 'No, you are not, I saw you having lunch just a few minutes ago'. How may an explanation of those facts go? In the first case, we may essay the following explanation: $A$ assertively uttered 'I'm hungry' because 'I'm hungry' means that the speaker is hungry, $A$ was hungry (and she knew it), and she deemed it relevant to convey the information that she was hungry because she wanted everyone to go out and have lunch. In the second case, we may essay the following explanation: $A$ rejected an assertive utterance of 'I'm hungry' made by $B$ because 'I'm hungry' means that the speaker is hungry, $A$ had good reasons to think that $B$ wasn't actually hungry ( $A$ saw $B$ eating a few minutes before), and $A$ deemed it relevant/convenient explicitly to contradict $B$ (maybe because she knows that $B$ finds $C$ s company unpleasant, and $A$ deems that getting the group to go out for 
lunch is a good way to avoid contact with $C$, and $A$ doesn't like $B$, so she wants her to be uncomfortable).

So, we may explain why a particular event of acceptance or rejection took place only by taking into account these kinds of considerations. And, using Horwich's model of explanation, we'll be able to assign high probability to an event of acceptance or rejection on a given situation only if we describe the situation in terms that make the acceptance or rejection highly likely-in particular, by appealing to considerations that go well beyond sentence meaning and sentential truth or falsity, and delve into the epistemic, the pragmatic and even the psychological. This is why, even if we regard acceptance and rejection facts as suitable explananda for a cluster of theories, speakers' intuitions will still be more directly relevant to semantic theorizing than facts of acceptance and rejection: fewer theories will be involved when confronting a truth-theoretic semantic theory with its evidential basis if we take intuitions to be the main evidence for or against a theory of meaning.

\section{References}

ALMOG, J., PERRY, J., WETTSTEIN, H. (eds.). Themes from Kaplan. Oxford: Oxford University Press, 1989.

CASO, R. “Assertion and relative truth". Synthese, 191, pp. 1309-1325, 2014. URL: http://link.springer.com/article/10.1007\%2Fs11229-013-0329-6

DAVIDSON, D. "Truth and meaning". Synthese, 17, pp. 304-323, 1967. URL: http://link.springer.com/article/10.1007\%2FBF00485035

GARCÍA-CARPINTERO, M., KÖLBEL, M. (eds). Relative Truth. Oxford: Oxford University Press, 2008.

HORWICH, P. Truth-Meaning-Reality. Oxford: Oxford University Press, 2010.

HORWICH, P. “An undermining diagnosis of relativism about truth". Mind, 123, pp. 733-752, 2014. URL:

http://mind.oxfordjournals.org/content/123/491/733

KAPLAN, D. "Demonstratives: an essay on the semantics, logic, metaphysics, and epistemology of demonstratives and other indexicals". In J. Almog, J.

Manuscrito - Rev. Int. Fil., Campinas, v. 38, n.2, pp.65-88, jul.-ago. 2015. 


\section{8[Ramiro Caso}

Perry and H. Wettstein (eds.) (1989), pp. 481-563.

KÖLBEL, M. "Two dogmas of Davidsonian semantics". The Journal of Philosophy, 98, pp. 613-635, 2001. URL: http://www.jstor.org/stable/3649462

KÖLBEL, M. “Truth in semantics”. Midwest Studies in Philosophy, 32, pp. 242-257, $2008 \mathrm{a}$.

KÖLBEL, M. "Motivations for relativism". In M. García-Carpintero and M. Kölbel (eds.) (2008), pp. 1-38, 2008b.

KÖLBEL, M. “The evidence for relativism”. Synthese, 166, pp. 375-395, 2009. URL: http://link.springer.com/article/10.1007\%2Fs11229-007-9281-7

MACFARLANE, J. Assessment Sensitivity: Relative Truth and Its Applications. Oxford: Clarendon Press, 2014. URL: http://johnmacfarlane.net/reltruth.pdf 